NO donor, possibly by formation of RNS. In addition, the optimum concentration for cardioprotection was different for each donor.

Funding This study was supported by the BBSRC and NiCox.

\section{GUANIDINOACETATE N-METHYLTRANSFERASE KNOCKOUT MICE EXHIBIT NORMAL LEFT VENTRICULAR REMODELLING, HAEMODYNAMICS AND SURVIVAL AFTER MYOCARDIAL INFARCTION DESPITE LACK OF PHOSPHOCREATINE}

doi:10.1136/hrt.2009.191064f

C A Lygate, D Dawson, D Medway, L Sebag-Montefiore, S Neubauer. Department of Cardiovascular Medicine, University of Oxford, Oxford, UK

Guanidinoacetate N-methyltransferase (GAMT) catalyses the final step of creatine biosynthesis such that $\mathrm{GAMT}^{-/-}$mice have undetectable levels of phosphocreatine and creatine and accumulation of the precursor (phospho-)guanidinoacetate (PGA). Like phosphocreatine, PGA acts as an energy reservoir, but energy transfer via creatine kinase is 100 times slower. We hypothesised that reduced energy transfer would be detrimental following myocardial infarction (MI). Methods $\mathrm{GAMT}^{-/-}$and wild-type controls received coronary artery ligation or sham operation ( $n=104)$, with 3D echocardiography and left ventricular haemodynamics after 6 weeks.

Results Sham GAMT $^{-1-}$ mice had reduced pressure-generating capacity compared with wild-type (wt), with left ventricular systolic pressure and $\mathrm{dP} / \mathrm{dt}_{\max }$ both significantly lower and impaired contractile reserve. Despite this, there was no significant difference in post-MI survival between $\mathrm{GAMT}^{-/-}$and wt. Both $\mathrm{GAMT}^{-/-}$and wt infarct groups exhibited left ventricular dilatation compared with sham controls, and systolic and diastolic function was also severely impaired. However, there was no significant difference between $\mathrm{GAMT}^{-1-}$ and wt infarct groups for left ventricular systolic pressure, left ventricular end-diastolic pressure, $\mathrm{dP} / \mathrm{dt}_{\max }$, or Tau, nor for end-diastolic and endsystolic volumes or ejection fraction. Left ventricular/body weight increased by $30 \%$ in $\mathrm{GAMT}^{-/-}$and $27 \%$ in wt, indicating a similar degree of left ventricular hypertrophy in response to MI.

Conclusions Loss of energy transfer in $\mathrm{GAMT}^{-/-}$mice was not detrimental to left ventricular remodelling, haemodynamics and survival post-MI. As acute reduction of energy transfer in the rat infarct model dramatically reduces survival, this strongly suggests that significant compensatory processes occur in $\mathrm{GAMT}^{-/-}$mice as a result of creatine loss during early life.

\section{THE TIME COURSE OF INORGANIC PHOSPHATE RELEASE AND ACTOMYOSIN ATPASE RATE IN PERMEABILISED CARDIAC TRABECULAE}

doi:10.1136/hrt.2009.191064g

C Mansfield, T West, D Bickham, M Ferenczi. National Heart and Lung Institute, Imperial College London, SAF Building, London, UK

The rate of inorganic phosphate $\left(\mathrm{P}_{\mathrm{i}}\right)$ release, and therefore the crossbridge ATPase rate, was determined in permeabilised rat trabeculae. Contraction was elicited by laser-flash photolysis of NPE-caged ATP, and time-resolved $\mathrm{P}_{\mathrm{i}}$ release was monitored using MDCC-PBP, a coumarin-labelled phosphate binding protein, which fluoresces upon $\mathrm{P}_{\mathrm{i}}$ binding. The ATPase rate during the first turnover of the total crossbridges (assuming $150 \mu \mathrm{M}$ myosin heads) was 23/s. The rate decreased to a steady state of $4 / \mathrm{s}$ after the eighth turnover $(0.5-0.6 \mathrm{~s}$ after activation). This rate is comparable to published values of 3-10/ $\mathrm{s}$, made $\sim 15 \mathrm{~s}$ after activation using a NADH-linked enzyme assay of ADP release. The advantage of using MDCC-PBP is that the control of mechanochemical coupling can be examined from the onset of force production. Force production and $\mathrm{P}_{\mathrm{i}}$ release were simulated using a seven-step scheme. Force was attributed to the states in the sequence A.M.ADP.P $\leftrightarrows$ A.M.ADP1 $\leftrightarrows$ A.M.ADP2, with strain sensitivity incorporated into the isomerisation of A.M.ADP. The A.M.ADP.P $P_{i}$ and A.M.ADP2 states populated rapidly as force was increasing. In contrast, the preisomerisation A.M.ADP1 accumulated slowly after the force plateau was reached and became the dominant force-bearing state at the time of the eighth crossbridge turnover. Experiments are ongoing to examine how the distribution of A.M states changes in response to rapid length changes.

\section{O20 DETERMINATION OF TROPONIN I PHOSPHORYLATION SITES IN HUMAN HEART MUSCLE}

doi:10.1136/hrt.2009.191064h

${ }^{1} \mathrm{~S}$ B Marston, ${ }^{1} \mathrm{~A}$ E Messer, ${ }^{1} \mathrm{C}$ E Gallon, ${ }^{2} \mathrm{R}$ Sancho-Sollis, ${ }^{2} \mathrm{~J}$ W Walker. ${ }^{1}$ Imperial College, London, UK; ${ }^{2}$ University of Arizona, Arizona, USA

It is well established that troponin I is a phosphoprotein. Phosphorylation alters its functional properties and this modulation of function through the action of kinases and phosphatases plays a role in tuning the contractile apparatus. The prime example of this is phosphorylation by protein kinase A (PKA) as part of inotropic and lusitropic responses to $\beta$-adrenergic stimulation. What is considerably less certain is the 'where' and 'when' of these phosphorylation processes in the human heart. Recent research has addressed this question with new techniques and the results have been surprising and somewhat disconcerting. Quantitative measurements of total phosphorylation by phosphate affinity sodium dodecylsulphate-polyacrylamide gel electrophoresis (SDSPAGE) indicate that $1.6 \mathrm{~mol}$ of $\mathrm{Pi}$ are incorporated per mole of troponin I in the donor heart. According to current literature, troponin I is phosphorylated in vitro by PKA at Ser22 and 23, by protein kinase C (PKC) at Ser41, Ser43 and Thr142 and by PAK1 and AMPK at Ser149. Both phosphate affinity SDS-PAGE and Fourier transform mass spectrometry plus ECD show that troponin I is mostly bis-phosphorylated and that three-quarters of the bisphosphorylated species is phosphorylated at Ser22 and 23, the PKA-specific sites. Somewhat surprisingly, there is no evidence of phosphorylation at Ser41, Ser43, Thr142 or Ser149 in human or rat heart muscle and the remaining phosphorylation is at Ser76 or Thr77 (the mass spectrometry techniques do not yet distinguish between the two). In end-stage failing heart muscle the level of phosphorylation is reduced to one-sixth of the donor level, therefore hypotheses that invoke PKC phosphorylation of troponin I need to be revised.

\section{PHENOTYPE OF THE ACTC E99K TRANSGENIC MOUSE REPRODUCES HYPERTROPHIC CARDIOMYOPATHY IN PATIENTS}

doi:10.1136/hrt.2009.191064

${ }^{1}$ W Song, ${ }^{2} \mathrm{D}$ J Stuckey, ${ }^{1} \mathrm{E}$ Dyer, ${ }^{1} \mathrm{D}$ Wells, ${ }^{1} \mathrm{~S} \mathrm{E}$ Harding, ${ }^{2} \mathrm{C}$ A Carr, ${ }^{2} \mathrm{~K}$ Clarke, ${ }^{1} \mathrm{~S}$ B Marston. Imperial College, London, UK; ${ }^{1}$ Oxford University, Oxford, UK

The mutation Gly99lys (E99K) in the cardiac actin (ACTC) gene has been found to cause hypertrophic cardiomyopathy (HCM) in 75 hypertrophic obstructive cardiomyopathy (HOCM) or LVNC patients. Transgenic mice expressing 50\% E99K mutant cardiac actin in their hearts were generated and studied. Over $30 \%$ male and $70 \%$ female E99K mice died between 28 and 45 days Anaesthetised 7-month-old male transgenic mice and their nontransgenic littermates were studied using in-vivo cine magnetic resonance imaging. Abnormal cardiac morphology and significantly lower ejection fractions and reduced stroke volumes were observed in the transgenic mice. Peak left ventricular ejection rates were reduced. Left ventricle function of 9-month-old female non-transgenic and transgenic mice were studied with an in-vivo conductance catheter. The transgenic mice had significantly reduced ejection fraction, increased end-diastolic pressure and impaired relaxation. Left ventricular dilation has been observed 